\title{
A empatia médica e a graduação em medicina
}

\author{
Medical empathy and graduation in medical school
}

\section{La empatía médica y la graduación en medicina}

\author{
Bruna C. Provenzano, André P. G. Machado," \\ Mary T. A. S. Rangel, Renata N. Aranha
}

\begin{abstract}
Resumo
O modelo biopsicossocial de assistência preconiza que, para que se tenha atenção integral, é essencial que haja uma relação médico-paciente sólida, tendo na comunicação seu alicerce. Nesse contexto, a empatia médica, um dos catalisadores desse processo comunicativo, é conceituada como uma adequada compreensão do paciente pelo médico e a capacidade de comunicar esta compreensão. É um conceito multidimensional, com quatro aspectos principais: emocional, moral, cognitivo e comportamental. Desde a metade do século XX, a empatia médica vem sendo considerada uma competência fundamental para o médico contemporâneo. Diante disso, surgiram diversos instrumentos que permitem sua avaliação e mensuração entre os estudantes e os profissionais de saúde. A Escala Jefferson é um dos principais exemplos, tendo sido aplicada em diversas universidades ao redor do mundo. De uma forma geral, os resultados mostraram dicotomia entre os dois gêneros, redução da empatia ao longo dos anos da formação médica e associação negativa com a escolha de especialidades tecnológicas pelo estudante. No Brasil, ainda há poucos estudos envolvendo essa questão. No I Censo MedUERJ, abrangendo os alunos de Medicina da Universidade do Estado do Rio de Janeiro, foi realizada a avaliação da empatia médica por meio dessa escala, corroborando a diferença entre homens e mulheres, porém não a sua redução ao longo da graduação, nem a disparidade quanto à opção por especialidades. Permanecem, portanto, controvérsias acerca do tema, que merecem esforços em seu esclarecimento, visto que, por se tratar de uma competência cognitiva, a empatia pode ser aprendida pelos estudantes, cabendo à escola médica a responsabilidade de ensiná-la.
\end{abstract}

Descritores: Empatia médica; Educação médica; Estudantes de medicina.

\begin{abstract}
The biopsychosocial healthcare model advocates that, in order to achieve an integral attention, it is essential to have a solid doctor-patient relationship with the communication as its' foundation. In this regard, medical empathy, one of the catalyzers of this communicative process, is defined as the suitable comprehension of the patient's emotions and the ability to communicate it. It is a multidimensional concept, with four main aspects: emotional, moral, cognitive and comportamental. Since mid-Twentieth century, medical empathy has been considered a fundamental competency for the contemporary physician. Therefore, various instruments that allow its evaluation and measurement in health students and professionals have been created. The Jefferson Scale is one of the main examples, and it has been applied in several universities all around the world. In general, the results showed a dichotomy between genders, a reduction of empathy throughout the years of medical formation and a negative association with the student's choice for a technological speciality. In Brazil, there are a few studies assessing this situation. In the I MedUERJ census, including the medical students of Universidade do Estado do Rio de Janeiro, the medical empathy was evaluated according to the Jefferson Scale, corroborating the difference between women and men. However, there has not been a significant decrease in medical empathy throughout the graduation years, nor a disparity between the speciality choices. In conclusion, the controversies still remaining around this subject deserve some effort in order to clarify them. Since it is a cognitive ability, it can be learned by the students, being a medical school responsibility to teach it.
\end{abstract}

Keywords: Medical empathy; Medical education; Medical students. 


\section{Resumen}

El modelo biopsicosocial de asistencia preconiza que, para que se proporcione atención integral, es fundamental la existencia de una relación médico-paciente solida que tenga en la comunicación su cimiento. En ese contexto, la empatía médica, uno de los catalizadores de este proceso comunicativo, se conceptualiza como una comprensión adecuada del paciente por el médico y la capacidad de comunicar esta comprensión. Es un concepto multidimensional, con cuatro aspectos principales: emocional, moral, cognitivo y conductual. Desde mediados del siglo XX, la empatía médica se ha considerado una competencia fundamental para el médico contemporáneo. En consecuencia, surgieron muchos instrumentos que permiten su evaluación y medición entre los estudiantes y los profesionales de salud. La escala de Jefferson es un buen ejemplo, habiendo sido aplicada en diversas universidades alrededor del mundo. De forma general, los resultados mostraron contrastes entre los dos géneros, reducción de la empatía a lo largo de los años de formación médica y asociación negativa con la elección de especialidades tecnológicas por parte del estudiante. En el Brasil, todavía hay pocos estudios desarrollando esa cuestión. En el I censo MedUERJ, que abarca a los alumnos de Medicina de la Universidad del Estado de Rio de Janeiro, se realizó la evaluación de la empatía médica por medio de esa escala, corroborando la diferencia entre hombres y mujeres, pero no la reducción a lo largo de la graduación, ni la disparidad en cuanto a la opción por especialidades. Por lo tanto persisten controversias acerca del tema, que merecen esfuerzos para su esclarecimiento, puesto que por tratarse de una competencia cognitiva, la empatía puede ser aprendida por los estudiantes, correspondiendo a la escuela médica la responsabilidad de enseñarla

Palabras clave: Empatía médica; Educación médica; Estudiantes de medicina.

\section{Introdução}

O termo empatia advém da palavra grega empatheia, a qual significa afeição. Foi utilizado pela primeira vez em 1904 pelo escritor Vernon Lee (1856-1935), para quem o processo empático correspondia à projeção da energia, atividades e sentimentos de cada indivíduo, equivalendo ao fenômeno psicológico Einfühlung, identificado pelo psicanalista alemão Theodor Lipps (1851-1914), seu contemporâneo. Ao longo do século XX, outros psicanalistas, como Carl Rogers (1902-1987), preconizaram que a empatia fosse vista de uma forma mais ampla, interpretando-a como um verdadeiro interesse pelo paciente. E mais: ela tornou-se um aspecto da própria personalidade do ser humano. ${ }^{1}$

A empatia médica é um conceito multidimensional, que engloba aspectos tanto nos campos emocionais e subjetivos quanto nos cognitivos e objetivos. Quatro dimensões básicas foram definidas: emocional, moral, cognitiva e comportamental. ${ }^{2}$ Dessa forma, empatia corresponderia à habilidade de imaginar os sentimentos do paciente (emocional), à motivação pessoal de ser empático (moral), à capacidade de identificar e entender as reações do paciente (cognitivo) e, por fim, à técnica de transmitir ao paciente essa compreensão (comportamental). A boa prática da medicina passa a ter na empatia médica uma

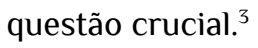

No ano de 2001, um grupo de pesquisadores do Jefferson Medical College observou que havia uma contradição nos estudos sobre empatia médica. Por um lado, este conceito se tornava cada vez mais central no desempenho profissional e na educação médica. Por outro, havia poucas pesquisas empíricas, uma persistente ausência de consenso sobre sua conceituação e nenhum instrumento que permitisse sua aferição da forma como desejavam. A partir desta demanda, foi desenvolvida a Escala Jefferson, o primeiro instrumento de avaliação de empatia elaborado especificamente para a medicina, o médico e o estudante de medicina, logo ganhando ampla aceitação e se tornando o mais utilizado em pesquisas sobre o tema no mundo. ${ }^{4}$

A Escala Jefferson considera a empatia predominantemente sob a ótica cognitiva. Ela é composta por 20 perguntas, que são respondidas pelo estudante, médico ou outro profissional de saúde em uma escala de Likert, cujos valores vão de um a sete, sendo que o um equivale a "discordo totalmente" e o sete "concordo totalmente". Ao término, realiza-se o somatório dos valores obtidos para cada pergunta. Desta forma, as pontuações mínima e máxima possíveis são, respectivamente, 20 e 140 pontos. Não há definição de um ponto de corte a partir do qual se considere ter ou não empatia desejável ou suficiente. O resultado obtido se baseia em uma gradação, ou seja, quanto 
maior a pontuação, mais empática é a pessoa. ${ }^{4}$

Em 2012, esse instrumento foi validado para o português brasileiro. Apesar de já haver a validação para o português de Portugal, existem diferenças culturais que poderiam comprometer a avaliação dos estudantes de medicina brasileiros. Diante das deficiências verificadas na assistência à saúde brasileira, tanto pública quanto privada, é imperioso o estudo da empatia em nossos cursos de graduação, buscando aprimorar a formação do médico. Não é sem razão que as diretrizes curriculares do nosso país indicam a empatia como uma competência a ser desenvolvida pelo aluno, seguindo uma tendência internacional. ${ }^{5}$

\section{Aspectos evolutivos da educação médica no século $X X$}

Ao se dirigir o olhar para o desenvolvimento da medicina ao longo da história da humanidade, percebe-se uma estreita relação entre os acontecimentos médicos e sociais. À medida que ocorrem profundas alterações na estrutura da sociedade, a medicina as acompanha, atualizando seus métodos diagnósticos, terapêuticos e de abordagem ao paciente. Nesse contexto, a descoberta de novas tecnologias e o progresso econômico exercem fortes pressões evolutivas sobre o modelo assistencial de saúde.

No início do século $X X$, frente às modificações dos fatores econômicos e às então recentes descobertas microbiológicas por Louis Pasteur e Robert Koch, Abraham Flexner propôs um novo modelo de percepção do conceito de saúde e doença, fortemente alicerçado em aspectos biomédicos. Com isto, foram estabelecidos quatro pilares fundamentais: 1) desenvolvimento de aulas teóricas baseadas somente na fisiopatologia das doenças; 2) fragmentação do indivíduo em sistemas; 3) relação médico-paciente centrada no médico; 4) ensino centrado na figura do professor. A partir da progressiva adoção deste modelo, a educação médica sofreu grandes mudanças, que se arraigaram através do tempo, persistindo com firmeza até o momento presente. O paciente passou a ter um papel secundário na educação médica, que se tornou centrada no modelo biomédico de adoecimento. ${ }^{6}$

Por um lado, o modelo flexneriano propiciou a organização e qualificação do ensino médico, indubitavelmente necessárias à época face à desestruturação reinante. Promoveu, em consequência, avanços científicos na área da saúde, possibilitando maior poder terapêutico e curativo às ciências médicas. Por outro lado, houve a perda da unidade do indivíduo e o declínio da relação médico-paciente. $\mathrm{O}$ afastamento entre o saber clínico e o cuidado resultou na deterioração da abordagem da pessoa enferma como sendo resultado de múltiplos fatores por ela vivenciados, em meio à sua realidade, ao seu ambiente. ${ }^{6}$

Posteriormente, os últimos 50 anos presenciaram substancial redução na confiança e no respeito à figura do médico por parte da sociedade, podendo-se atribuir isso, em grande parte, à falência deste modelo flexneriano, que atribui importância somente ao aspecto científico do ser humano. A crise do modelo biomédico advém da interpretação de que doença é definida meramente por parâmetros somáticos. Assim, não convém ao médico a preocupação com aspectos biopsicossociais do paciente. Seguindo esta linha, as doenças mentais chegaram a ser vistas como inexistentes por, de modo geral, não possuírem evidência de disfunção orgânica identificável. Neste mesmo contexto, doença era definida como uma alteração da normalidade anatômica ou bioquímica do organismo. Portanto, invariavelmente, o paciente era identificado como doente essencialmente por apresentar anormalidades em parâmetros laboratoriais e outros exames complementares, sem que fosse levado em conta aquilo que ele realmente sentia.

Como o que se pode considerar uma reação a essa conjuntura, surge um novo modelo de compreensão do indivíduo, da saúde e da doença: o modelo biopsicossocial. Este considera que, para uma abordagem completa ao paciente, é fundamental que sejam levados em conta os seus aspectos tanto psicológicos quanto sociais. A missão do médico agora é dar atenção tanto à disforia quanto ao distúrbio clínico que leva o paciente a buscar auxílio. Indo mais além, a disforia deve ser encarada não apenas como mera consequência da enfermidade, mas também como sua possível causa. ${ }^{8}$

O desafio desse novo modelo é criar um diálogo entre a medicina cientificista e o caráter assistencialista, permitindo a descoberta da pessoa e encontrando as origens tanto do sofrimento 
quanto da doença. Em outras palavras, a descoberta do corpo através do ser. ${ }^{6}$

\section{A incorporação da empatia médica ao exercício profissional}

O modelo biopsicossocial exige do novo profissional novas habilidades cujo objetivo é a melhor compreensão sobre o paciente. A relação médico-paciente torna-se uma questão central, sendo fundamentada na credibilidade, na confiabilidade e na responsabilidade, de modo a aumentar as chances de se obter sucesso diagnóstico e terapêutico. ${ }^{9}$ A capacidade de se estabelecer uma adequada comunicação entre as partes é de inquestionável importância, tornando-se alicerce para a construção da confiança e da credibilidade. ${ }^{8}$ A comunicação é um fenômeno social que envolve diversos aspectos semiológicos e antropológicos. Assim sendo, a empatia torna-se componente essencial para que haja a completa conquista da competência comunicativa por parte do médico. ${ }^{10}$

Surgiram então novas tendências curriculares que objetivaram redirecionar o enfoque hospitalocêntrico do modelo flexneriano. Estas buscaram associar o lado prático instituído neste modelo ao enfoque em promoção da saúde e prevenção das doenças, resultando na redefinição do perfil do profissional de saúde desejado. Naquele momento de novos rumos curriculares nascentes, houve reorientação no sentido de uma formação generalista do médico, incorporando preceitos éticos, visando à integralidade da assistência.?

A empatia facilita a comunicação entre médico e paciente, consolidando a relação entre ambos. Com isso, o paciente sente-se mais capaz para explicitar queixas e preocupações, favorecendo o aperfeiçoamento da anamnese. Como resultante, há o desenvolvimento de diagnósticos mais precisos. Além disso, o médico se torna apto a transmitir de forma mais eficaz seus conhecimentos ao paciente, contribuindo para a compreensão deste acerca da sua doença, gerando maior adesão ao tratamento e, por conseguinte, maior sucesso terapêutico. Portanto, ambos se sentem satisfeitos com os resultados, aprimorando a qualidade de vida do paciente ao mesmo tempo em que aumenta a sensação de realização profissional do médico. A contribuição da empatia chega até a redução do número de processos judiciais contra os médicos, cuja origem central é a insuficiente comunicação entre estes e seus pacientes."

A Associação Americana de Universidades Médicas (em inglês, Association of American Medical Colleges - AAMC) incluiu, em diretrizes publicadas em 1998, o desenvolvimento da empatia em sua relação de objetivos para a graduação em medicina, vistos os diversos benefícios que essa competência traz à prática médica. A empatia logrou, dessa forma, transformar-se em questão primordial da atividade médica, o que gerou a necessidade de contemplá-la com uma melhor avaliação e, mais, com um método que permitisse a sua mensuração."

\section{A avaliação da empatia médica durante o curso de graduação}

Nos Estados Unidos, durante a última década, diversos estudos foram publicados utilizando a Escala Jefferson. Dentre os resultados obtidos com os estudantes de graduação em medicina, despertaram especial atenção três aspectos: 1) verificou-se declínio da empatia médica ao longo dos anos de formação profissional, destacando-se a diferença entre o terceiro e o quarto anos; 2) o sexo feminino alcançou escores mais elevados do que o masculino; 3) tanto os estudantes quanto os médicos que escolheram especialidades ditas orientadas para o paciente, como clínica médica, pediatria ou ginecologia, demonstraram possuir escores maiores do que aqueles que escolheram áreas mais especializadas, "voltadas para a tecnologia". 12,13

As avaliações empreendidas nas universidades americanas revelaram, portanto, que durante a própria graduação há uma redução da empatia dos estudantes, evidenciando uma deficiência notável na educação médica dos EUA. Esse resultado foi obtido tanto em estudos transversais quanto em estudos longitudinais prospectivos. ${ }^{13}$ Parece haver forte correlação entre o momento de queda da empatia e o início da prática clínica pelo estudante, no terceiro ano do curso. Algumas hipóteses foram levantadas na tentativa de explicar este fato, correlacionando-o tanto ao currículo médico da graduação quanto aos aspectos afetivo-emocionais dos alunos.

Em relação à formação escolar, haveria ambientes de aprendizado inadequados, em que o 
estudante sofreria humilhações e desprezo por parte dos professores. Além disso, os primeiros contatos entre o aluno e o paciente se fazem acompanhados por provas e testes de habilidades práticas, os quais nem sempre fornecem experiências agradáveis. Numa outra vertente, ocorre simultaneamente o excesso de horas de estudo e trabalho, contribuindo para o surgimento de estresse e depressão. De fato, o aluno vê suas expectativas confrontadas pela realidade, a qual nem sempre corresponde a seu idealismo prévio. Como se não bastasse, há também o isolamento social e familiar, devido à dedicação quase exclusiva que a graduação médica impõe ao estudante."

Em outros países, alguns dos resultados obtidos não foram concordantes com os dos estudos americanos. De uma forma geral, as mulheres obtiveram escores mais elevados do que os homens, mantendo a dicotomia entre gêneros observada nos Estados Unidos. Contudo, no estudo polonês ${ }^{14}$ e no italiano ${ }^{15}$, não houve diferença estatisticamente significativa entre os sexos. Além disso, nem todos os estudos mostraram a queda da empatia ao longo da graduação, como se pôde verificar com os desenvolvidos em Portugal, ${ }^{16}$ Itália ${ }^{15}$ e Japão. ${ }^{17}$ Não obstante, em ao menos um caso, um estudo iraniano, não só a empatia aumentou ao longo dos anos, como também houve associação positiva entre o início da prática clínica e os escores obtidos, reforçando a falta de universalidade destes achados. ${ }^{18}$

Outro dado interessante são os próprios valores numéricos dos escores obtidos em cada população analisada. De um modo geral, os países ocidentais apresentaram valores maiores do que os países orientais. Este resultado foi discutido nos estudos japonês, coreano e iraniano, e todos correlacionam essa variação não a uma efetiva menor empatia, mas sim às diferenças culturais entre esses países, assim como às diferentes formas de ingresso no curso de graduação. ${ }^{17,18,19}$

No Brasil, os poucos estudos realizados na área da empatia médica empregaram basicamente metodologias qualitativas. Em parte talvez como consequência de que, enquanto a discussão sobre a relação médico-paciente se mostra relativamente avançada nos Estados Unidos, ocorrendo há pelo menos cinquenta anos, em nosso país esta começou a receber a merecida atenção somente a partir da década de $1990 .^{2}$

\section{A empatia médica no I Censo MedUERJ}

A avaliação da empatia médica, utilizandose a escala Jefferson, foi incluída no I Censo MedUERJ, realizado no final de 2011, na Faculdade de Ciências Médicas (FCM) da Universidade do Estado do Rio de Janeiro (UERJ). Este estudo foi desenvolvido através da aplicação de um questionário on-line aos alunos de graduação que compunham o quadro da FCM-UERJ, excluídos os cinco alunos $(0,9 \%)$ que se encontravam com sua matrícula trancada, assim como os quatro alunos $(0,7 \%)$ que tiveram o seu ingresso no curso por transferência ex officio. Todos os 542 alunos aptos responderam ao questionário.

Nossos alunos apresentaram um escore médio para empatia de 121,8, que superou os encontrados em diversos estudos, como o iraniano, cuja média foi de 105,1 (embora seus autores ainda o tenham considerado alto), ${ }^{18}$ e mesmo, embora mais discretamente, o original americano quando da elaboração do instrumento, com seus 120,0 pontos. $^{12}$

No que tange ao sexo, a tendência verificada na maioria dos estudos foi corroborada, com escores de 123,4 e 119,4 para os sexos feminino e masculino, respectivamente. Não encontramos relação entre escolha da especialidade a seguir e a empatia.

Sem sombra de dúvida, a questão mais controversa envolvendo a empatia dos estudantes de medicina é o possível declínio no decorrer do curso de graduação, em especial a partir do terceiro ano, justamente quando se inicia de forma mais consistente o contato do aluno com o paciente. Todavia, um expressivo número de trabalhos não identificou tal declínio, e os nossos dados acompanharam este último grupo: nossos alunos não demonstraram perder empatia durante sua trajetória na faculdade, o que teremos que analisar à luz de suas próprias particularidades e de nossas características curriculares.

\section{Conclusão}

Acima de qualquer questionamento reside a importância do estudo da empatia médica que, embora já venha se desenvolvendo lentamente nas últimas décadas, parece despertar crescente 
interesse. ${ }^{20}$ Em primeiro lugar, pela relevância de que desfruta como requisito primordial para a relação médico-paciente, ${ }^{21}$ incluindo os efeitos positivos a ela creditados em relação a diversos desfechos clínicos, ${ }^{22}$ assim como a possibilidade de ser trabalhada com perspectiva de aprimoramento. ${ }^{23}$ Associa-se a isso o rol substantivo de limitações, dúvidas e controvérsias que ainda envolvem o tema, ${ }^{24,25}$ e que parecem não encontrar, por ora, indícios de superação. ${ }^{26}$

Os resultados revelados no censo que realizamos somam-se aos diversos aspectos ainda indefinidos no campo da empatia médica, cujos estudos, ao redor do mundo, seguem tentando perscrutar. Não obstante, tais dados devem contribuir para que possamos formar um perfil de como se comporta a empatia médica na formação dos estudantes do nosso país.

\section{Referências}

1. Hayward R. Historical Keywords: Empathy. The Lancet. 2005;366(9491):1071 http://dx.doi.org/10.1016/ S0140-6736(05)67407-7

2. Gelhaus P. The desired moral attitude of the physician: (III) care. Med Health Care Philos. 2013;16(2):125-39. http://dx.doi.org/10.1007/s11019012-9380-1

3. Stepien KA, Baernstein A. Educating for empathy: a review. J Gen Intern Med. 2006;21(5):524-30.

4. Hojat M, Mangione S. Jefferson Scale of Physician Empathy. Health Policy Newsl. 2001;14(4).

5. Paro HBMS, Daud-Gallotti RM, Tibério IC, Pinto RMC, Martins MA. Brazilian version of the Jefferson Scale of Empathy: psychometric properties and factor analysis. BMC Med Educ. 2012;12:73. http:// dx.doi.org/10.1186/1472-6920-12-73

6. Halperin EC, Perman JA, Wilson EA. Abraham Flexner of Kentucky, his report, Medical Education in the United States and Canada, and the historical questions raised by the report. Acad Med. 2010;85(2):203-10. http://dx.doi.org/10.1097/ ACM.0b013e3181c88506

7. Ludmerer KM. Commentary - understanding the Flexner report. Acad Med. 2010;85(2):193-6. http:// dx.doi.org/10.1097/ACM.0b013e3181c8f1e7

8. Engel GL. The need for a new medical model - a challenge for biomedicine. Science. 1977;196(4286):129-36.

9. Schraiber LB. O médico e seu trabalho: limites da liberdade. São Paulo: Hucitec; 1993.
10. Halpern J. What is clinical empathy?. J Gen Intern Med. 2003;18(8):670-4. http://dx.doi.org/10.1046/ j.1525-1497.2003.21017.x

11. Neumann M, Edelhäuser F, Tauschel D, Fischer MR, Wirtz M, Woopen C, et al. Empathy decline and its reasons - a systematic review of studies with medical students and residents. Acad Med. 2011;86(8):996-1009. http://dx.doi.org/10.1097/ACM. 0b013e318221e615.

12. Hojat M, Gonnella JS, Nasca TJ, Mangione S, Vergare M, Magee M. Physician Empathy: Definition, Components, Measurement, and Relationship to Gender and Specialty. Am J Psychiatry. 2002;159(9):1563-9.

13. Hojat M, Mangione S, Nasca TJ, Rattner S, Erdmann JB, Gonnella JS, et al. An empirical study of decline in empathy in medical school. Med Educ. 2004;38(9):934-41

14. Kliszcz J, Nowicka-Sauer K, Trzeciak B, Nowak P. Empathy in health care providers - validation study of the Polish version of the Jefferson Scale of Empathy. Adv Med Sci. 2006;51:219-25

15. Di Lillo M, Cicchetti A, Loscalzo A, Taroni F, Hojat M. The Jefferson Scale of Physician Empathy: Preliminary Psychometrics and Group Comparisons in Italian Physicians. Acad Med. 2009;84(9):1198-202. http://dx.doi.org/10.1097/ACM.0b013e3181b17b3f.

16. Aguiar P, Salgueira A, Frada T, Costa Mj. Empatia médica: tradução, validação e aplicação de um instrumento de medição. Atas do X Congresso Internacional Galego-Português de Psicopedagogia. Braga: Universidade do Minho; 2009.

17. Kataoka Hu, Koide N, Ochi K, Hojat M, Gonnella JS. Measurement of empathy among Japanese medical students: psychometrics and score differences by gender and level of medical education. Acad Med. 2009;84(9):1192-7. http://dx.doi.org/10.1097/ ACM.0b013e3181b180d4

18. Rahimi-Madiseh M, Tavakol M, Dennick R, Nasiri J. Empathy in Iranian medical students - a preliminary psychometric analysis and differences by gender and year of medical school. Med Teach. 2010;32(11):e4718. http://dx.doi.org/10.3109/0142159X.2010.509419

19. Suh DH, Hong JS, Lee DH, Gonnella JS, Hojat M. The Jefferson Scale of Physician Empathy: A preliminary psychometric study and group comparisons in Korean physicians. Med Teach. 2012;34(6):e464-8. http:// dx.doi.org/10.3109/0142159X.2012.668632.

20. Burcher P. Emotional intelligence and empathy: its relevance in the clinical encounter. Patient Intell. 2011;3:23-8. http://dx.doi.org/10.2147/PI.S11070

21. Baykan Z, Naçar M, Demirel SO. Evaluation of empathic skills and tendencies of medical students. Acad Psychiatry. 2011;35(3):207-8.

22. Del Canale S, Louis DZ, Maio V, Wang X, Rossi G, 
Hojat M, et al. The relationship between physician empathy and disease complications: an empirical study of primary care physicians and their diabetic patients in Parma, Italy. Acad Med. 2012;87(9):1243-9.

23. Hojat, M. Ten approaches for enhancing empathy in health and human services cultures. J Health Hum Serv Adm. 2009;31(4):412-50.

24. Pedersen R. Empathy: a wolf in sheep's clothing? Med Health Care Philos. 2008;11(3):325-35.

25. Lelorain S, Brédart A, Dolbeault S, Sultan S. A systematic review of the associations between empathy measures and patient outcomes in cancer care. Psychooncology. 2012;21(12):1255-64. http://dx.doi. org/10.1002/pon.2115

26. Looi JC. Empathy and competence. Med J Aust. 2008;188(7):414-6.

\section{Bruna C. Provenzano}

Faculdade de Ciências Médicas. Universidade do Estado do Rio de Janeiro. Rio de Janeiro, RJ, Brasil.

\section{André P. G. Machado}

Departamento de Clínica Médica. Faculdade de Ciências Médicas. Universidade do Estado do Rio de Janeiro. Rio de Janeiro, RJ, Brasil.

\section{Mary T. A. S. Rangel}

Departamento de Tecnologias da Informação e Educação em Saúde. Faculdade de Ciências Médicas. Universidade do Estado do Rio de Janeiro. Rio de Janeiro, RJ, Brasil.

\section{Renata N. Aranha}

Departamento de Ginecologia e Obstetrícia. Faculdade de Ciências Médicas. Universidade do Estado do Rio de Janeiro. Rio de Janeiro, RJ, Brasil. 